\title{
Contrasting patterns of niche partitioning between two anaerobic terminal oxidizers of organic matter
}

\author{
Brian B Oakley ${ }^{1}$, Franck Carbonero ${ }^{1}$, Scot E Dowd ${ }^{2}$, Robert J Hawkins ${ }^{3}$ and Kevin J Purdy ${ }^{1}$ \\ ${ }^{1}$ University of Warwick, School of Life Sciences, Coventry, UK; ${ }^{2}$ Research and Testing Laboratories, \\ Lubbock, TX, USA and ${ }^{3}$ University of Reading, Department of Biological Sciences, Reading, UK
}

\begin{abstract}
Understanding the ecological principles underlying the structure and function of microbial communities remains an important goal for microbial ecology. We examined two biogeochemically important taxa, the sulfate-reducing bacterial genus, Desulfobulbus, and the methanogenic archaeal genus, Methanosaeta, to compare and contrast niche partitioning by these two taxa that are ecologically linked as anaerobic terminal oxidizers of organic material. An observational approach utilizing functional gene pyrosequencing was combined with a community-based reciprocal incubation experiment and characterization of a novel Desulfobulbus isolate. To analyze the pyrosequencing data, we constructed a data analysis pipeline, which we validated with several control data sets. For both taxa, particular genotypes were clearly associated with certain portions of an estuarine gradient, consistent with habitat or niche partitioning. Methanosaeta genotypes were generally divided between those found almost exclusively in the marine habitat ( $\sim 30 \%$ of operational taxonomic units (OTUs)), and those which were ubiquitously distributed across all or most of the estuary ( $\sim 70 \%$ of OTUs). In contrast to this relatively monotonic distribution, for Desulfobulbus, there were many more genotypes, and their distributions represented a wide range of inferred niche widths from specialist genotypes found only at a single site, to ubiquitous or generalist genotypes found in all 10 sites examined along the full estuarine gradient. Incubation experiments clearly showed that, for both taxa, communities from opposite ends of the estuary did not come to resemble one another, regardless of the chemical environment. Growth of a Desulfobulbus isolated into pure culture indicated that the potential niche of this organism is significantly larger than the realized niche. We concluded that niche partitioning can be an important force structuring microbial populations, with biotic and abiotic components having very different effects depending on the physiology and ecology of each taxon.
\end{abstract}

The ISME Journal (2012) 6, 905-914; doi:10.1038/ismej.2011.165; published online 24 November 2011

Subject Category: microbial population and community ecology

Keywords: methanogenesis; niche partitioning; pyrosequencing; sulfate reduction

\section{Introduction}

Sulfate-reducing bacteria (SRB) and methanogenic archaea (MA) perform an important step of the carbon cycle, the remineralization of organic matter in anaerobic environments. SRB and MA both utilize small carbon compounds resulting from the degradation of complex organic matter and are of global biogeochemical importance-SRB and MA may account for up to half of carbon mineralization in marine and freshwater environments, respectively (Jorgensen, 1982; Lovley et al., 1982), and comprise the majority of subseafloor sediment communities (D'Hondt et al., 2002) that may account for up to onethird of the earth's total biomass (Whitman et al., 1998). Although the potential metabolic niche space

Correspondence: BB Oakley. Current address: Agricultural Research Service, USDA, Richard Russell Research Center, 950 College Station Road, Athens, GA 30605, USA.

E-mail: brian.oakley@ars.usda.gov

Received 16 June 2011; revised 19 September 2011; accepted 3 October 2011; published online 24 November 2011 of SRBs includes facultative acetogenic and syntrophic lifestyles (Plugge et al., 2011), the ecological relationship of SRB and MA in anaerobic environments canonically depends on the availability of sulfate; where sulfate concentrations or recycling rates are high, sulfate reduction is usually a dominant process, where sulfate is limiting, methanogenesis generally predominates (Laanbroek and Veldkamp, 1982; Lovley and Klug, 1983; Widdel, 1988).

To compare and contrast the ecology of MA and SRB, MA in the genus Methanosaeta provide a useful model of a strict metabolic specialist as they utilize acetate as their sole electron donor, electron acceptor and carbon source (Patel and Sprott, 1990). Methanosaeta are thus particularly susceptible to competition from other acetate-utilizing MA (Jetten et al., 1990) and SRB (Ward and Winfrey, 1985). In contrast, SRB in the genus Desulfobulbus are able to couple the respiration of sulfate to the oxidation of three-carbon compounds and can also grow fermentatively, mixotrophically or by using nitrate or nitrite as an alternative electron acceptor (Stams, 1984; Lien et al., 1998; Sass et al., 2002; 
Suzuki et al., 2007). Because of their geochemical importance, natural ecological linkage and contrasting metabolic flexibility, Desulfobulbus and Methanosaeta represent useful model taxa to analyze microbial distribution patterns and some basic aspects of microbial ecology such as niche partitioning. In this study, we focus on niche partitioning by each of these genera and combine observational and experimental approaches to infer how niche-based processes may influence community structure.

Environmental niche specialization has been shown to be an important force structuring microbial communities (West and Scanlan, 1999; Johnson et al., 2006; Hunt et al., 2008), and, in the same estuarine gradient studied here, genotypes of Desulfobulbus have been shown to be sequentially distributed (Hawkins and Purdy, 2007; Oakley et al., 2010) in a manner similar to original descriptions of the phenomenon of niche differentiation (May and Macarthur, 1972). In contrast, for Methanosaeta, evidence for cosmopolitan distributions of 16S rRNA genotypes has been reported (Carbonero, 2010). Until recently, however, accurate determination of microbial niche occupancy has been hindered by chronic undersampling of clone libraries, or limited resolution of fingerprinting techniques such as denaturing gradient gel electrophoresis or terminal restriction fragment length polymorphisms. We utilized recent advances in sequencing technology (Margulies et al., 2005; Hamady et al., 2008) to provide a solution to this problem and combined environmental sequencing with experimental manipulations and cultivation to achieve our main objectives of comparing and contrasting the patterns and mechanisms of niche occupancy of Methanosaeta and Desulfobulbus. Because of the relative novelty of pyrosequencing and debates about its proper applications to assess microbial diversity (Huse et al., 2010; Kunin et al., 2010), we also developed and validated a data analysis pipeline, which can rapidly analyze pyrosequencing data.

\section{Materials and methods}

Study site and sample collection

Triplicate sediment cores for pore water analyses and DNA extractions were taken at low tide from 11 sites along the Colne estuary in September 2004 as previously described (Hawkins and Purdy, 2007). Samples for the slurry experiment were collected in September 2008 from sites 1 and 10, representing the marine and freshwater ends of the estuary, respectively.

\section{Molecular methods}

Amplification of $\operatorname{dsr} B$ and $\operatorname{mcr} A$ genes. Amplification of a portion of the bisulfite reductase $(d s r \mathrm{~B})$ gene targeted to Desulfobulbus was achieved by PCR with the primers DSR458F (5'-CGCBCTGAT GRATCTGTG-3'; Oakley et al., 2010) and 2060F'
(5'-CAACATCGTYCATACMCAGGG-3'; modified from (Geets et al., 2006) to improve specificity) in combination with DSR4R (5'-GTGTAGCAGTTACCGCA-3'; Wagner et al., 1998) as previously described (Oakley et al., 2010). New primers specifically and sensitively targeting Methanosaeta mcrA genes were designed using sequences from the functional gene pipeline and repository (http://fungene.cme.msu. edu/). Based on maximum-likelihood phylogenetic reconstructions performed in ARB (Ludwig et al., 2004), a monophyletic clade was identified as a target group containing sequences from environmental samples and the three described Methanosaeta species M. concilii, M. harundinacea and M. thermophila. Using a program, THERMOPHYL, we wrote for this purpose (Oakley et al., 2011), we designed new primer pairs F1 (5'-CTACATGTCCG GYGGTGTC-3') and R1 (5'-TAGTTRGCGCCYCTC AKCTC- $\left.3^{\prime}\right)$, and F2 (5'-GTCGGWTTCACMCAGTA CGC- $\left.3^{\prime}\right)$ and R2 (5'- TGCCCTCGTCKGACTGGTA-3'), which were identified as optimally specific and sensitive (Oakley et al., 2011) and subsequently used for empirical testing.

Methanosaeta-specific morA primer assessments. Primer specificity, sensitivity and amplification efficiency were evaluated empirically using genomic DNA from $M$. concillii (DSM6752), M. harundinacea (DSM 17206), M. thermophila (DSM4774), Methanosarcina mazei (DSM2053), Methanosarcina acetovorans (DSM2834) and environmental DNA from Colne Estuary sediments as previously described (Oakley et al., 2011). DNA was extracted from actively growing cultures using the DNeasy Blood and Tissue kit (Qiagen, Crawley, UK) and environmental DNA was extracted from sediment samples as previously described (Purdy, 2005).

Following validation of the PCR sensitivity and specificity, the same protocol was used to amplify three technical replicates from each of three biological replicates (sediment samples taken within a $50 \mathrm{~cm}$ radius at each site). Products were cleaned using the QiaQuick Gel Extraction kit (Qiagen) and pooled after normalization based on quantification of PCR products using QuantIt PicoGreen (Invitrogen, Paisley, UK) as per manufacturers instructions and fluorescence measured with a Perkin-Elmer (Cambridge, UK) Wallac Victor ${ }^{2} 1420$ plate reader.

Pyrosequencing methods. Pyrosequencing was performed at Research and Testing Laboratory (Lubbock, TX, USA: http://www.researchandtesting.com). Using tagged amplicon methods similar to those described previously (Dowd et al., 2008) modified for titanium chemistry (Roche, Branford, CT, USA). Twenty cycles of PCR were utilized $\left(94^{\circ} \mathrm{C}\right.$ for $30 \mathrm{~s}, 50^{\circ} \mathrm{C}$ for $30 \mathrm{~s}$ and $72{ }^{\circ} \mathrm{C}$ for $40 \mathrm{~s}$ ) with a final extension at $72{ }^{\circ} \mathrm{C}$ for 10 min to incorporate the linkers and tag A 200 flow Titanium sequencing run was performed according to Roche protocols with amplicon signal processing. Following the sequencing and image processing, the 
sequences were binned into individual multi-fasta files and used for data analysis.

\section{Pyrosequencing analysis}

Initial quality control. Raw sequence data were first edited and screened using a series of custom Perl and Bioperl scripts, which performed the following initial steps as per the recommendations of Kunin et al. (2010) and Huse et al. (2007): trimming of pyrosequencing tag sequences, screening for presence of PCR primer sequences and removal of sequences with one or more ambiguous base calls. Based on expected amplicon sizes and sequence length frequency distributions, sequences were limited to a range of 375-385 bp for $d s r \mathrm{~B}$ and $411-419 \mathrm{bp}$ for mcrA. Sequences passing these screens were then subjected to several additional quality control measures by translations in all three forward frames and screens for the presence of conserved amino-acid motifs. Sequences in the appropriate frame passed the screen and trailing nucleotides were trimmed to produce complete codons.

Methodological validation. To distinguish errors inherent to the pyrosequencing itself versus analytical errors, we pyrosequenced single colonies and compared the results of several analytical approaches. First, cell-suspension PCR was performed from single axenic colonies of a Desulfobulbus strain we isolated from the freshwater end of the estuary and Methanosaeta concilii (DSM6752) using the $d s r \mathrm{~B}$ and morA PCR protocols, respectively, as described above. Next, pyrosequence data from these PCR products were compared with a reference sequence determined by bidirectional Sanger sequencing to determine a baseline error rate inherent to pyrosequencing. Pairwise comparisons were performed with a perl script using Needle-Wasserman global alignments as implemented in the EMBOSS package (Rice et al., 2000) with gap-extension and gap-open parameters set to 2 and 14, respectively. Subsequently, errors introduced by commonly used multiple sequence alignment (MSA) strategies were assessed by aligning a random subset of 5000 pyrosequences using the programs mothur (Schloss et al., 2009), MUSCLE (Edgar, 2004) and MAFFT (Katoh et al., 2002). The alignment error for each sequence was calculated as the absolute difference in percent identity for that sequence as aligned to the reference sequence in the pairwise alignments above versus the MSA. Finally, the effect of the alignment errors on operational taxonomic unit (OTU) designations was determined by clustering sequences into OTUs using mothur. To compare these results to an alternative MSA-independent method, sequences were also clustered using CD-HIT-EST (Li and Godzik, 2006).

Clustering and data analysis pipeline. Sequences were grouped into similarity clusters or OTUs, by
CD-HIT-EST (Li and Godzik, 2006) with parameters set to the default recommendations except for the -g parameter, which was set to search all clusters to place a sequence into the most similar cluster, rather than the first cluster which meets the similarity threshold. The output from CD-HIT provided the input for a data analysis pipeline we constructed, which parses the clustering results, reformats the data for input into mothur and links a series of scripts in R ( $R$ Development Core Team, 2010) to produce graphical and statistical summaries of the data according to desired sampling unit. More information and the open-source code for the pipeline can be found at http://go.warwick.ac.uk/ thermophyl/pipeline. Representative sequence data have been submitted to GenBank under accession numbers: JN684079-JN684183.

\section{Phylogenetic and statistical analyses}

Sequences which passed all the screens described above were aligned as nucleotides to curated seed databases based on amino-acid alignments (http:// fungene.cme.msu.edu/) and incorporated into ARB (Ludwig et al., 2004). Trees were built with maximum-likelihood and neighbor-joining algorithms in ARB and with MrBayes (Huelsenbeck and Ronquist, 2001) with the following modifications to default parameters: GTR model of evolution with gammadistributed rate variation, number of chains set to six, numbers of swaps to three and temperature to $0.1 ; 200000$ generations produced stable convergence. Phylogenetic clustering was assessed with UniFrac (Lozupone and Knight, 2005) and Parsimony tests (Martin, 2002), which compare observed phylogenies with a null model derived from random permutations.

\section{Slurry experiment}

Sediments from the endpoints of the estuary (sites 1 and 10) were incubated in a factorial design in which sulfate and salinity were manipulated independently. For each site, three replicate sediment cores $(0-5 \mathrm{~cm}$ depth) were taken within $50 \mathrm{~cm}$ of each other, and maintained at $8{ }^{\circ} \mathrm{C}$ for $<12 \mathrm{~h}$ before initiating the experiment. Each core was homogenized and $0.4-0.5 \mathrm{~g}$ of sediment added to anaerobic Balch tubes containing $5 \mathrm{ml}$ of medium in one of four formulations $(\mathrm{S}+/ \mathrm{Cl}+, \mathrm{S}+/ \mathrm{Cl}-, \mathrm{S}-/ \mathrm{Cl}+, \mathrm{S}-/$ $\mathrm{Cl}-$ ) with sulfate and chloride concentrations based on pore water concentrations at sites 1 and 10. DSMZ Medium 194 was prepared anaerobically and $\mathrm{Na}_{2} \mathrm{SO}_{4}, \mathrm{NaCl}$, and $\mathrm{MgCl}_{2}$ added in the amounts shown in Table 1. Carbon was provided to all treatments as sodium propionate per standard media formulation $\left(1.5 \mathrm{gl}^{-1} ; 15.6 \mathrm{mM}\right)$. Propionate is degraded to acetate in sediment slurries (Purdy, 1997), which can subsequently be used by Methanosaeta under some circumstaces. DNA was extracted according to (Purdy et al., 1996) from 
Table 1 Details of sulfate and chloride additions for the reciprocal incubation experiment

\begin{tabular}{llrc}
\hline \multirow{2}{*}{ Treatment } & \multicolumn{3}{c}{ Sulfate and chloride addition $\left(\mathrm{gl}^{-1}\right)$} \\
\cline { 2 - 4 } & $\mathrm{Na}_{2} \mathrm{SO}_{4}$ & $\mathrm{NaCl}$ & $\mathrm{MgCl}_{2}$ \\
\hline S+/Cl+ & 3.0 & 20.0 & 3.1 \\
S+/Cl- & 3.0 & 1.0 & 0.4 \\
S-/Cl+ & 0.075 & 20.0 & 3.1 \\
S-/Cl- & 0.075 & 1.0 & 0.4 \\
\hline
\end{tabular}

Additions were made to DSMZ Medium 194 based on pore water concentrations at sites 1 and 10 as described in the text.

sediment samples at the initiation of the experiment and after incubation at $23{ }^{\circ} \mathrm{C}$ for 7 days. Data were analyzed using the cca routine of the vegan package (Oksanen, 2010) in R (R Development Core Team, 2010).

Desulfobulbus cultivation methods

Fresh sediment samples were transferred anaerobically into enrichment media (DSMZ Medium 194) prepared as described above in liquid enrichments and agar plates. Cultures were screened for Desulfobulbus by PCR with the primers dsr458F (Oakley et al., 2010) and dsr4R (Wagner et al., 1998) and FISH using the probes EUB338 (Amann et al., 1990), DSBB228 (Loy et al., 2002), DSBB660 (Loy et al., 2002), and a newly designed probe designated Dsb1447 (5'-CTGCCCCCTCGAAAGGTT-3') hybridized with $20 \%$ formamide according to standard FISH protocols (Fuchs et al., 2007). Positive enrichments were transferred until an axenic culture was obtained.

\section{Results and discussion}

Pyrosequencing analysis pipeline development and validation

Based on PCR from a single colony, the error rate of pyrosequencing itself was quite low (Supplementary Figure S1A), with a mean error rate $<0.5 \%$ for both genes, consistent with previous reports (Huse et al., 2007). Some of this error reflects errors incorporated during PCR; error rates with the proofreading polymerase, Pfx (Invitrogen), were indeed lower (mean of $0.10 \%$; data not shown), but we deliberately chose to focus on error rates using protocols with Taq and nested PCR because these are often required to achieve adequate amplification from environmental DNA.

The quality of MSA is a critical but often overlooked step in determining the accuracy and reproducibility of many sequencing projects. We found that alignment errors, as distinguished from pyrosequencing errors, can produce a significant overestimation of species richness and diversity. In this study we measured alignment errors ranging from $0.25 \%$ to $>1.5 \%$ (Supplementary Figure S1B). Because alignment errors disproportionately occurred in the distal $\left(3^{\prime}\right)$ end of the alignment, we appended an anchoring $18 \mathrm{bp}$ oligo sequence (5'-ACCACA CAAAAACCCACA-3') to both ends of each sequence to see if this improved the alignment, but it did not. Even with sequences screened to a narrow length range (370-375 bp), we still observed similar alignment errors.

To see how the accumulation of these errors affected the clustering of sequences into OTUs, we next compared several methods of designating OTUs. Using Muscle with default parameters to align a control data set of 5000 sequences obtained from a single axenic colony as described above, 43 OTUs were observed, 15 of which were singletons (Supplementary Figure S1C). Based on pairwise Needle-Wasserman alignments of each singleton sequence to each sequence in the original data set, 13 of these were designated false singletons (Supplementary Figure S1C). Using Muscle with optimized parameters resulted in fewer OTUs (29), fewer initial singletons (5) and fewer false singletons (3), but the best performance was achieved with the progam CD-HIT-EST, which uses a MSA-independent method of clustering sequences and produced 12 OTUs with three true singletons (Supplementary Figure S1C). Because the greatest pairwise sequence difference strictly attributable to pyrosequencing error was below the $3 \%$ cutoff (Figure 1a) used to group sequences into OTUs, we can infer that some classification errors arose from the CD-HIT algorithm, but clearly it is more conservative than even the best MSA-based approach tested. As the control data set should represent a single OTU, conservative equated with best performance in this case. When applied to sequences from a complex community, it is possible that CD-HIT may underestimate the true diversity, but this seems unlikely given these results. For MSA, even with the small control data set (5000 sequences) used here, accurate alignment is often problematic; for the much larger data sets characteristic of most modern sequencing projects, MSA-based approaches can be intractable.

Based on these results and because CD-HIT also has the important advantage of being computationally efficient, we used CD-HIT to cluster sequences and developed an analysis pipeline to allow for automatic downstream processing of the sequence data. After clustering using the pipeline, a series of scripts in $\mathrm{R}$ (R Development Core Team, 2010) automatically produce graphical and statistical summaries and comparisons of the data based on user-defined sample groups. Additionally, we developed the pipeline to also allow for use of the popular program mothur (Schloss et al., 2009) without having to cluster sequences based on MSA distance matrices.

\section{Analysis of environmental mcrA and $d s r B$ pyrosequences}

Comparisons to previous data sets and technical replicates. Because pyrosequencing has been shown 

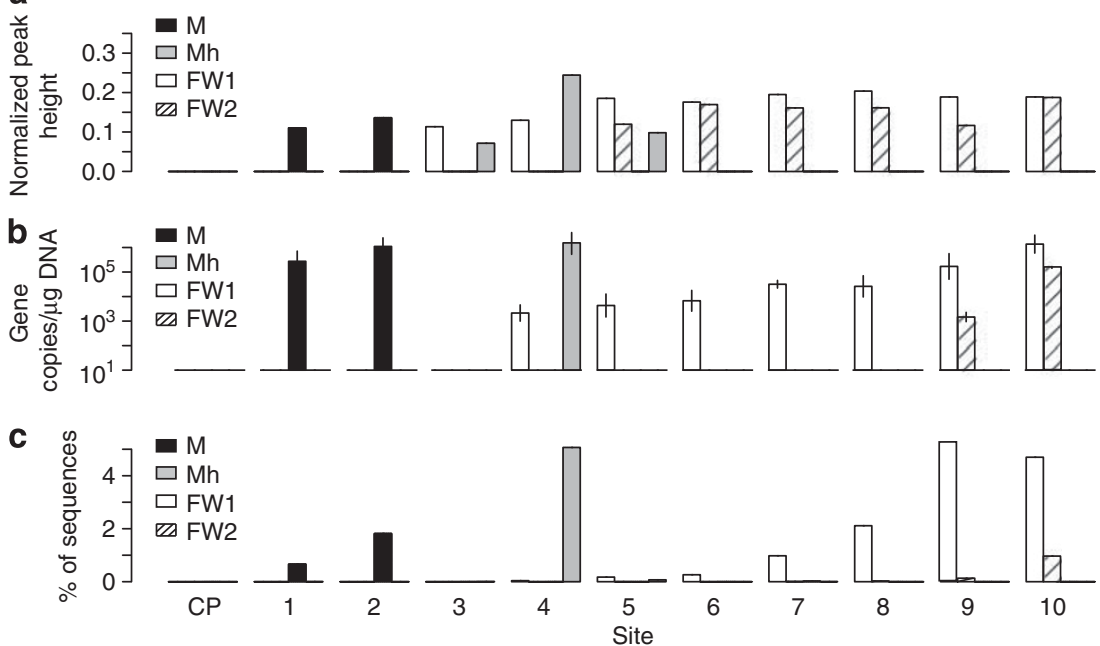

Figure 1 Comparison of pyrosequencing results (c) to denaturing gradient gel electrophoresis (a) and quantitative-PCR (qPCR) (b) as determined previously (Oakley et al., 2010) to survey the distribution of four Desulfobulbus genotypes. For the pyrosequencing results (c) the data set of ca 33000 sequences was queried for exact matches to qPCR assays; Y-axis represents the percentage of pyrosequences corresponding to each taxon. Genotype designations are M, Marine; Mh, Mesohaline; FW1, Freshwater1; FW2, Freshwater 2. Figure is modified from Purdy (2010).

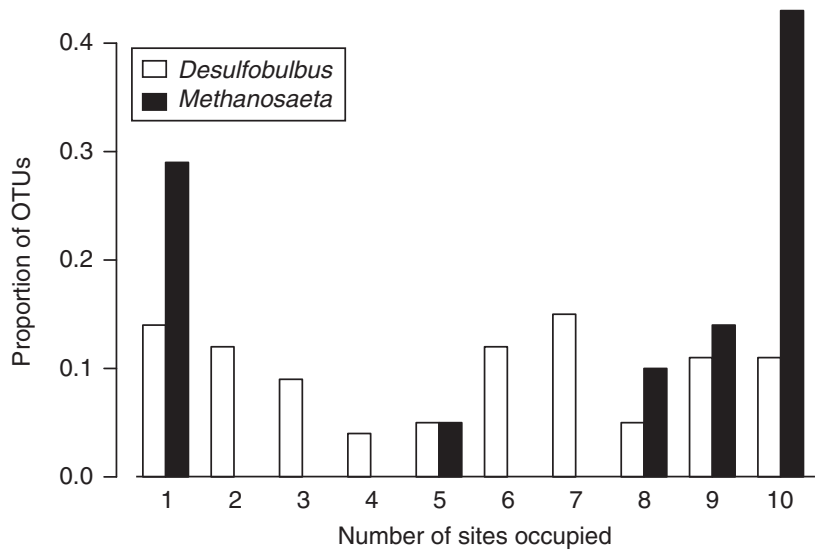

Figure 2 Species-occupancy distributions for Desulfobulbus and Methanosaeta. showing the number of sampling sites occupied by genotypes of each taxon.

to preferentially sequence certain templates, we followed recent recommendations (Gomez-Alvarez et al., 2009) to run multiple technical replicates for amplicon sequencing. Analysis of OTU distributions across three technical replicates of both the mcrA and $d s r \mathrm{~B}$ sequencing revealed largely similar results (Supplementary Figure S2). For further analyses, data from all three technical replicates were pooled to reduce technical variations. Summaries of richness and diversity parameters for the pooled sequence data are shown in Supplementary Figure S3.

As an additional test of the validity of the pyrosequencing and of our analysis pipeline applied to environmental samples, we compared the results with abundance measures of the spatial distributions of four Desulfobulbus genotypes we had determined previously using gradient gel electrophoresis and TaqMan quantitative-PCR (Oakley et al., 2010).

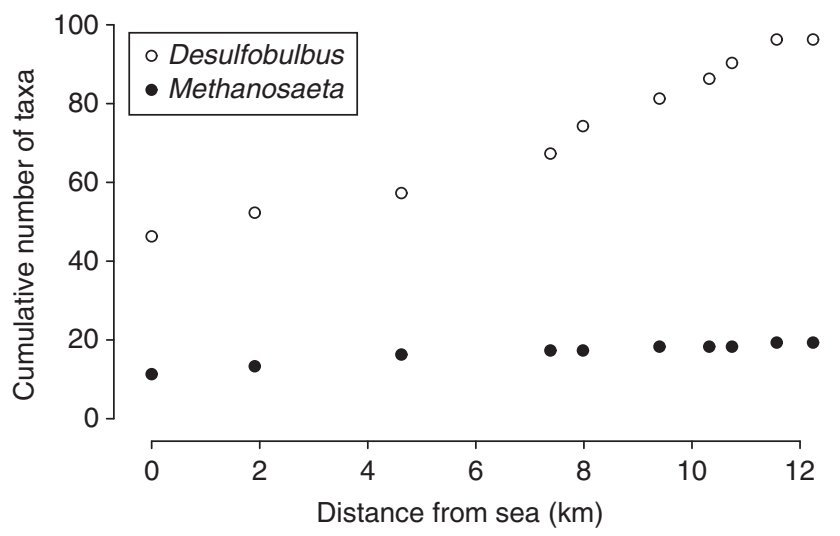

Figure 3 Species-area curves for Desulfobulbus and Methanosaeta. Y-axis represents the cumulative number of OTUs as determined with CD-HIT as described in the methods.

The pyrosequencing results were consistent with both of these methods, with only minor differences among the three (Figure 1). With all three methods, clear distinctions could be made among genotypes associated with certain portions of the estuary-a marine $(\mathrm{M})$ genotype, a mesohaline $(\mathrm{Mh})$ genotype, a widely-distributed freshwater genotype (FW1) and a freshwater genotype (FW2) with a more restricted distribution (Figure 1). These patterns are similar to data collected from con-generic bird species (Macarthur, 1958), which helped to establish niche theory in the field of ecology based on the inference that the distribution of taxa in space reflects optimization of an $n$-dimensional space of biotic and abiotic variables.

Species-occupancy patterns. To give additional insights into the distribution patterns of Desulfobulbus 

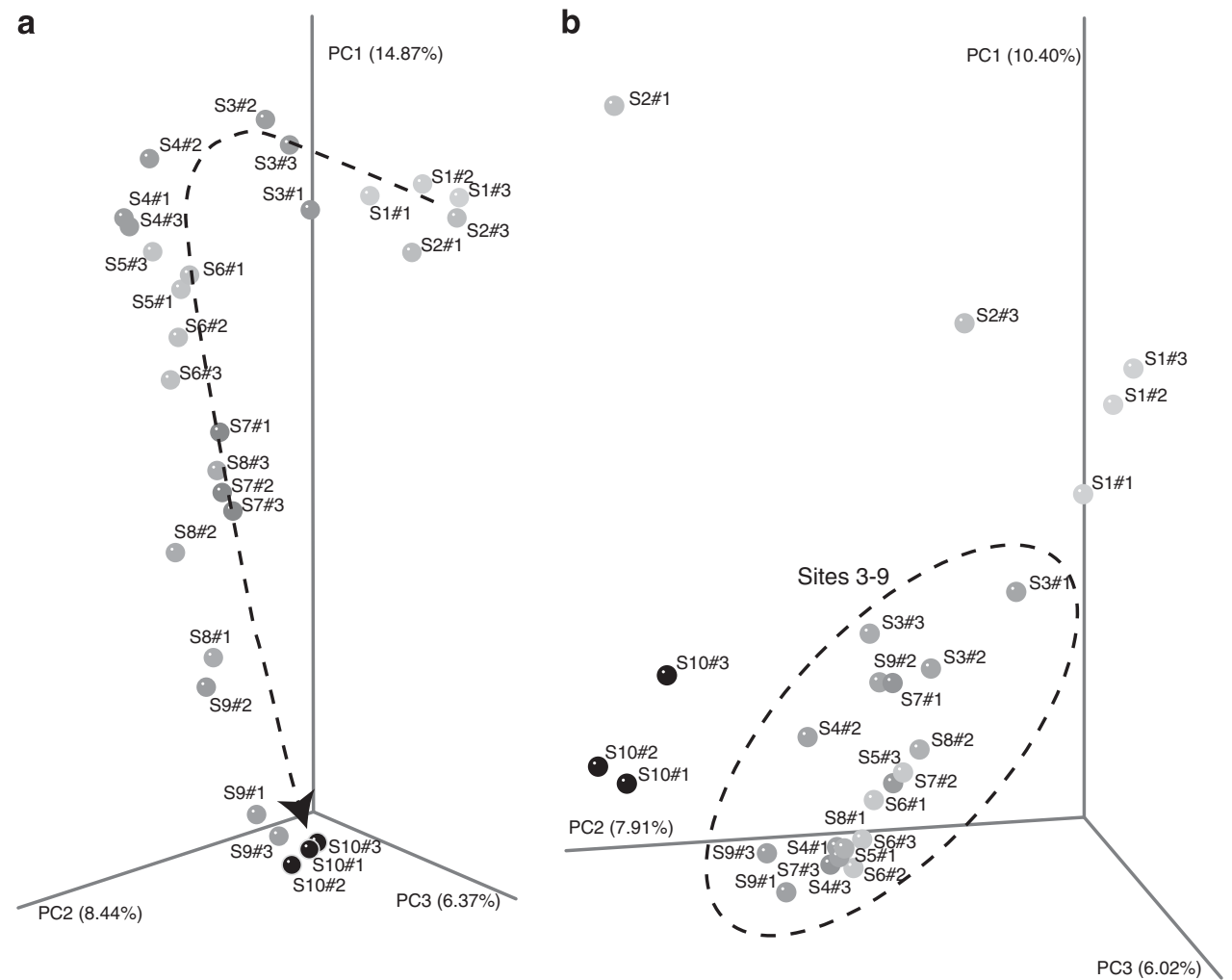

Figure 4 Phylogenetic clustering of sample sites based on PCA of phylogenetic position of each sequence as determined by UniFrac for Desulfobulbus (a) and Methanosaeta (b). Sequences were aligned and neighbor-joining trees containing all sequences constructed for each taxon as fully described in the methods. Points represent biological replicates for each sample site.

and Methanosaeta, we next performed a speciesoccupancy analysis (He and Gaston, 2003) to determine the number of sampling sites occupied by each taxon (Figure 2). Two conclusions were clear from this analysis. First, most Methanosaeta genotypes (ca 2/3) were found at many sites (eight or more) but nearly all the rest (ca 1/3) were found only at one site; these were the marine genotypes (Figure 2). Second, in contrast to the bimodal frequency distribution of Methanosaeta, Desulfobulbus genotypes were relatively evenly distributed across the range of what can be interpreted as 'specialist' genotypes restricted to a single site and 'generalist' genotypes encountered in all 10 sites (Figure 2). Nearly 60\% of Desulfobulbus genotypes were present at between two to seven sites (Figure 2). Based on these results, we concluded that Desulfobulbus genotypes can represent a full range of niche-occupancy patterns, from specialist to generalist, whereas Methanosaeta are generally either found across nearly the whole length of the estuary (generalists) with some genotypes restricted to a single site (specialists).

Species-area relationships. If Desulfobulbus does indeed occupy more niche spaces than Methanosaeta, a logical prediction is that more genotypes should be encountered when the same geographic area is sampled. Such relationships, known as species-area curves, have a long history as a description of beta diversity and as a means of understanding the ecology of an organism and its adaptations to different habitats (Arrhenius, 1921; $\mathrm{He}$ and Legendre, 1996). For Desulfobulbus and Methanosaeta, a comparison of species-area curves revealed dramatic differences-starting from the marine end of the estuary, Desulfobulbus has more genotypes than Methanosaeta, and novel genotypes are continually encountered as one moves up the estuary (Figure 3). In contrast, Methanosaeta populations are relatively depauperate and nearly all the genotypes present in the estuary have already been encountered simply by sampling the marine end of the estuary (Figure 3). These patterns obviously reflect the taxonomic definition chosen-we used a sequence-similarity cutoff of $85 \%$ for both taxa (chosen to roughly represent sequence divergence of these functional genes among described species), but other cutoffs also produced essentially similar results. Although these data don't reveal the processes underlying this pattern, they do provide further evidence for what can be viewed as the finescale niche adapation of Desulfobulbus relative to Methanosaeta.

Population distribution patterns. To better understand the association between particular sequence types and sampling site in the estuary, we next performed a cluster analysis based on the phylogenetic position of sequences by sampling site. Based on the distribution of sequence types along the 
estuarine gradient, for Desulfobulbus, it is clear that there is significant spatial autocorrelation of sequence types-that is, sequences originating from samples close to one another in the estuary tend to be more similar to one another than they are to sequences originating from more distant samples (Figure 4). For both taxa, there are clearly distinct clusters of sequence types representing 'marine' and 'freshwater' genotypes, but for Desulfobulbus, there was a clear gradient of genotypes that were distributed in a nearly linear fashion along the estuary (Figure 4a). Desulfobulbus genotypes from the two end points of the estuary were clearly distinct from one another and genotypes from intermediate positions in the estuary could also be distinguished as distinct clusters distributed in a strong gradient (Figure 4a). In contrast, for Methanosaeta, the main distinction was between estuarine and marine genotypes from the two end points of the estuary as distinct from all other locations, which clustered quite closely to one another (Figure 4b).

Taken together, these contrasting patterns of distribution suggest different models of niche differentiation for these two taxa. Although our data cannot distinguish among various evolutionary mechanisms such as drift or homologous recombination, or the importance of biogeographic factors such as dispersal, colonization and extinction, it is clear from the regular partitioning of Desulfobulbus that it has evolved to occupy a wide range of niche spaces from ubiquitously distributed (generalists) to narrowly distributed (specialists) in contrast to Methanosaeta in which the primary distinction is between marine and estuarine populations. It is possible to make several very general assumptions that perhaps shed some light on these differences between the two taxa. Based on calculations of reported doubling times for Desulfobulbus of around $10 \mathrm{~h}$ (Kuever et al., 2005) versus up to 12 days for Methanosaeta (Patel, 2001), we can roughly estimate the approximate number of generations that have inhabited the Colne estuary since the last glaciation as circa 13 million for Desulfobulbus and circa 45000 for Methanosaeta. This roughly 300-fold difference may contribute to the wide range of niche occupancy for Desulfobulbus relative to Methanosaeta.

\section{Experimental confirmations of patterns}

To better assess whether the differences in distributional patterns for these two taxa simply reflect environmental conditions at the time of sampling or evolutionary divergence of populations adapted to conditions at a particular geographic location, we next performed a reciprocal incubation experiment in which sediments from either end of the estuary were incubated in growth media mimicking both native and contrasting conditions. We used pyrosequencing to compare the communities at the two endpoints of the estuary to determine if one community would come to resemble the other given the appropriate environment. It did not (Figure 5).

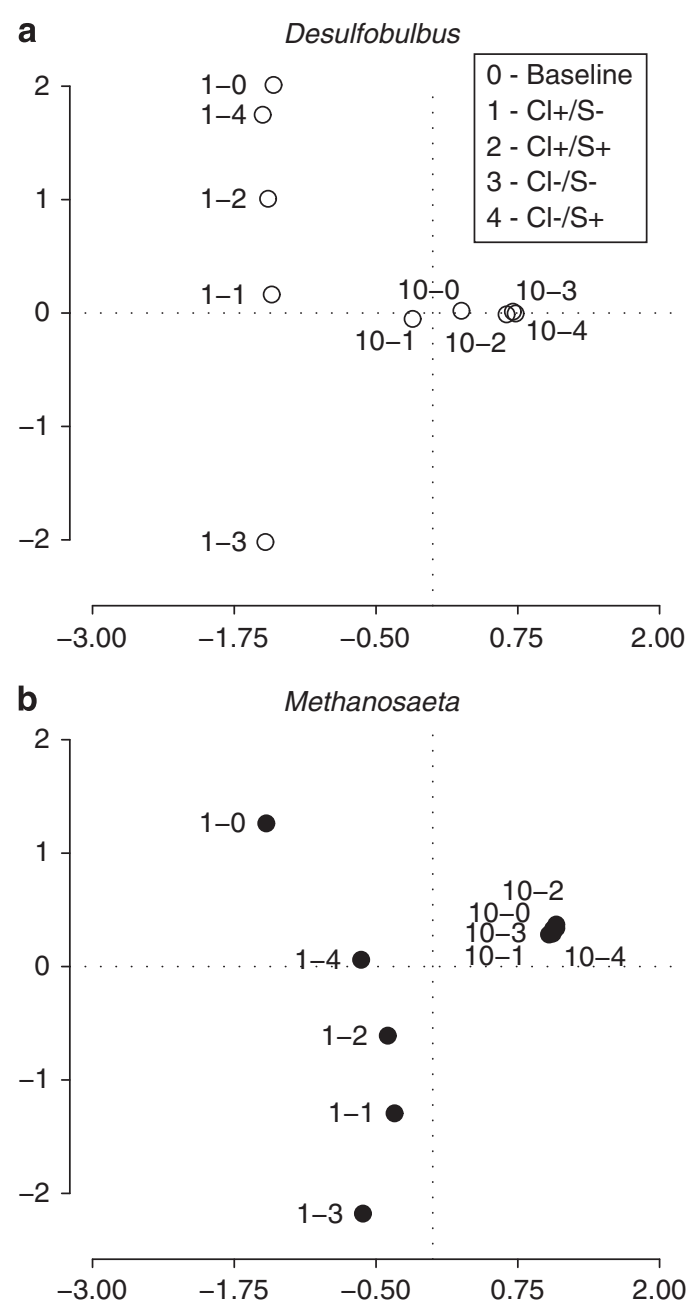

Figure 5 Response of (a) Desulfobulbus and (b) Methanosaeta populations to sediment slurry reciprocal incubation experiment. Graphs represent CCA results based on OTU distributions calculated from pyrosequencing with $d s r \mathrm{~B}$ and mcrA primers as described in the methods. Sampling site (1 or 10) is designated before the dash; experimental treatments are designated after the dash as shown in the legend. Axes represent first two eigenvectors from each CCA. Exact media formulations are given in Table 1.

In fact, for both taxa, the results were remarkably similar-populations from site 1 were very responsive to environmental conditions, whereas those from site 10 remained largely unchanged, and populations from sites 1 and 10 remained clearly distinct regardless of environmental conditions (Figure 5). Factors other than salinity and sulfate can of course affect these communities, but for two other potentially significant variables, temperature and organic carbon availability, there is no substantial variation across the $15 \mathrm{~km}$ of this estuary (Dong et al., 2006). The results of the experiment support a biogeographic hypothesis that populations in the sediments at these two sites are so different that they will not come to resemble the other site, regardless of environment.

Realized versus potential niche space. To further understand the factors determining niche space for 
a

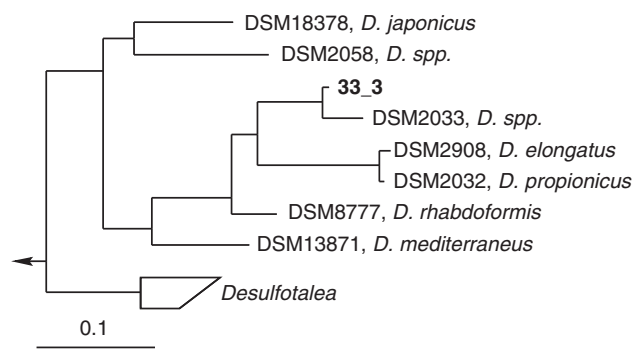

b

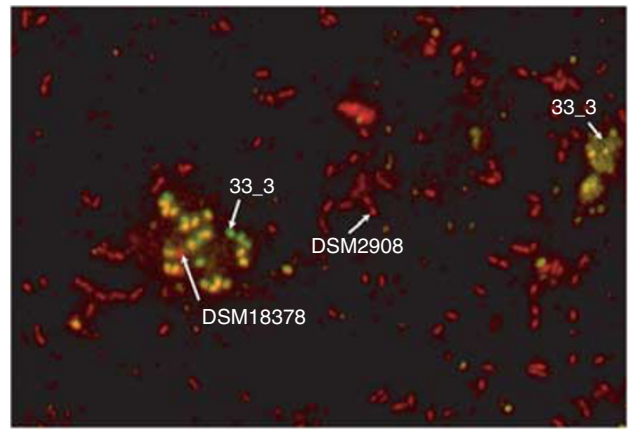

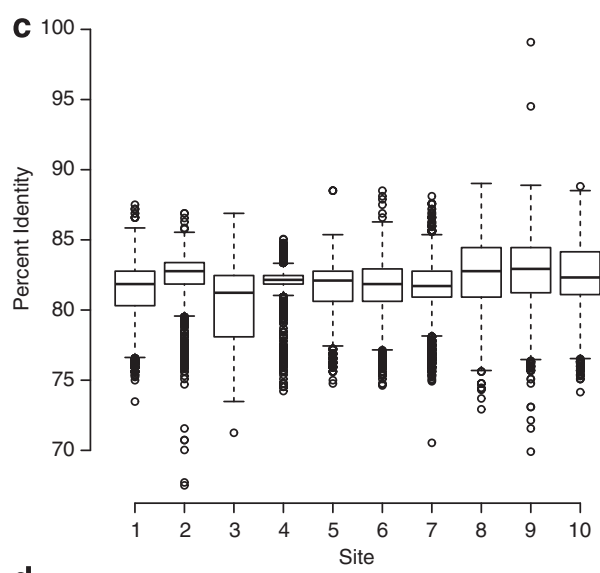

d

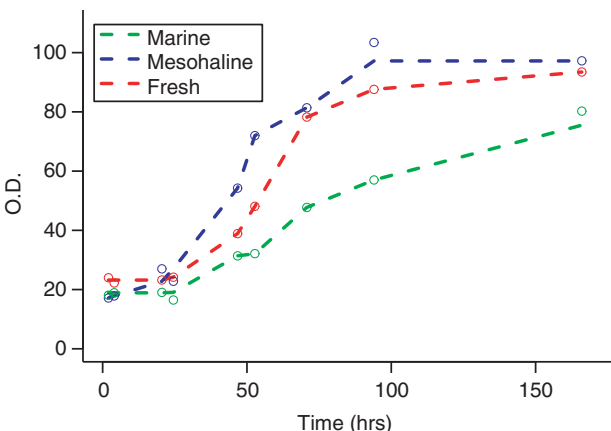

Figure 6 Natural distribution and growth medium preferences of a novel isolate designated 33_3 recovered from the Colne estuary. (a) Phylogenetic position of 33_3 relative to reference Desulfobulbus spp. based on sequences of the same region of the $d s r B$ gene used for pyrosequence analysis. (b) 33_3 grown with D. elongatus (DSM2908) and D. japonicus (DSM18378) and shown by fluorescence in situ hybridization with the probes Dsbb660-Cy3 and a newly-designed probe Dsb1447-Alx488 to target 33_3 as described in the methods. (c) Natural distribution of 33_3 sequence type in the Colne estuary as determined by pairwise sequence comparison with all $d s r$ B pyrosequences. For boxplots, horizontal bars indicate mean, boxes enclose 25th-75th percentile of data, whiskers indicate 10th-90th percentiles and circles indicate outliers of pairwise comparisons of 33_3 dsrB sequence with pyrosequences from each site. (d) Growth curves of 33_3 in marine, mesohaline and freshwater media. Media formulations were based on DSMZ Medium 194 as described in the text and shown in Table 1 , with marine medium equivalent to the $\mathrm{S}+/ \mathrm{Cl}+$ treatment, freshwater medium equivalent to $\mathrm{S}+/ \mathrm{Cl}-$ and mesohaline medium with intermediate concentrations of $\mathrm{NaCl}$ and $\mathrm{MgCl}_{2}$.

these taxa, we used cultivation as an alternative and complementary approach to our observational and experimental uses of pyrosequencing. An axenic culture of Desulfobulbus (termed '33_3') was recovered from site 10 using anaerobic enrichment techniques in liquid and solid media (Figures 6a and b). We were also able to cultivate Methanosaeta from the Colne estuary (Carbonero et al., 2010), but the slow growth rates and clumping growth habits of these archaeal isolates made experimental manipulations difficult.

We were first interested in understanding the natural distribution of the Desulfobulbus isolate and so queried our pyrosequencing data set for sequence similarity to the $d s r$ sequence of 33_3. Intriguingly, we found that 33_3 is quite an unusual sequence type that was sparsely represented in the pyrosequencing survey-only a single sequence had $>95 \%$ nucleotide similarity to 33_3 as determined by pairwise global alignments (Figure 6c). This single best match had five nucleotide mismatches (out of ca $400 \mathrm{bp}$ ) to the 33_3 dsr sequence, and interestingly, all of these were synonymous muta- tions. Although 33_3 was isolated from site 10 sediment collected in 2007 , we only found it at site 9 in the pyrosequencing data from sediment collected in 2004. This either reflects undersampling of the pyrosequencing, or more likely, slight temporal shifts in distribution such as those described previously (Hawkins and Purdy, 2007; Oakley et al., 2010). Regardless, it is clear that the distribution of 33_3 is quite narrow.

Taking this distribution as a direct measure of the realized niche space of 33_3, we then compared this with the potential environmental niche space as measured by growth in media representing a gradient of estuarine conditions (Figure 6d). Clearly, the realized niche is quite a bit smaller than the potential niche-replicate experiments showed that strain 33_3 actually grows slightly better in mesohaline media than freshwater media (Figure 6d). Although the exact mechanisms restricting niche space and their relative influences remain unclear, the distribution of these taxa appears to represent a dynamic combination of abiotic and biotic factors. 


\section{Conclusions}

The results of our pyrosequencing data were consistent with previous approaches based on denaturing gradient gel electrophoresis, Sanger sequencing of clone libraries and quantitative-PCR (Hawkins and Purdy, 2007; Carbonero, 2010; Oakley et al., 2010). However, because pyrosequencing provided several orders of magnitude more data than previously obtained, we were also able to properly employ a species-occupancy analysis to better understand the ecology of our model taxa. Our data reiterate the utility of pyrosequencing for microbial ecology, provided proper care is taken in the treatment and analysis of the data. In particular, we found MSA to be a crucial source of errors, and so chose to cluster our sequences with CD-HIT (Li and Godzik, 2006) to avoid both the computational challenges and potential for analytical errors introduced by MSA.

The majority of Methanosaeta sequences were cosmopolitan whereas the majority of Desulfobulbus sequences were restricted to particular sampling sites. Reciprocal-incubation experiments showed that sediment communities from opposite ends of the estuary differ independently of the chemical environment, consistent with biogeographic predictions. Experiments with a novel Desulfobulbus isolate demonstrated that the realized niche is substantially more constrained than the potential niche, consistent with ecological niche constraints as experienced by macroorganisms.

\section{Acknowledgements}

This study is part of the Marie Curie Excellence Grant for Teams project, MicroComXT, (MEXT-CT-2005-024112) funded under FP6. RJH was supported by the UK Natural Environment Research Council. We thank Tom Lie, Martin Ostrowski, Andy Millard and Kevin Gaston for helpful discussions.

\section{References}

Amann RI, Binder BJ, Olson RJ, Chisholm SW, Devereux R, Stahl DA. (1990). Combination of $16 \mathrm{~S}$ rRNA-targeted oligonucleotide probes with flow cytometry for analyzing mixed microbial populations. Appl Environ Microbiol 56: 1919-1925.

Arrhenius O. (1921). Species and area. J Ecol 9: 95-99.

Carbonero F. (2010). Distributions of Model Microorganisms Along an Estuarine Gradient. Department of Biological Sciences, University of Warwick: Coventry, p 151.

Carbonero F, Oakley BB, Purdy KJ. (2010). Improving the isolation of anaerobes on solid media: the example of the fastidious Methanosaeta. J Microbiol Methods 80: 203-205.

Dong LF, Nedwell DB, Stott A. (2006). Sources of nitrogen used for dentrification and nitrous oxide formation in sediments of the hypernutrified Colne, the nutrified Humber, and the oligotrophic Conwy estuaries, United Kingdom. Limnol Oceanography 51: 545-557.

Dowd SE, Sun Y, Wolcott RD, Domingo A, Carroll JA. (2008). Bacterial tag-encoded FLX amplicon pyrosequencing
(bTEFAP) for microbiome studies: bacterial diversity in the ileum of newly weaned Salmonella-infected pigs. Foodborne Pathog Dis 5: 459-472.

D’Hondt S, Rutherford S, Spivack AJ. (2002). Spivack, Metabolic activity of subsurface life in deep-sea sediments. Science 295: 2067-2070.

Edgar RC. (2004). MUSCLE: a multiple sequence alignment method with reduced time and space complexity. $B M C$ Bioinformatics 5: 113.

Fuchs BM, Pernthaler J, Amann R. (2007). Single cell identification by fluorescence in situ hybridization. In: Reddy CA et al. (eds). Methods for General and Molecular Microbiology. ASM Press: Washington, DC, pp 886-896.

Geets J, Borremans B, Diels L, Springael D, Vangronsveld J, van der Lelie D et al. (2006). DsrB gene-based DGGE for community and diversity surveys of sulfatereducing bacteria. J Microbiol Methods 66: 194-205.

Gomez-Alvarez V, Teal TK, Schmidt TM. (2009). Systematic artifacts in metagenomes from complex microbial communities. ISME J 3: 1314-1317.

Hamady M, Walker JJ, Harris JK, Gold NJ, Knight R. (2008). Error-correcting barcoded primers for pyrosequencing hundreds of samples in multiplex. Nat Methods 5: 235-237.

Hawkins RJ, Purdy KJ. (2007). Genotypic distribution of an indigenous model microorganism along an estuarine gradient. FEMS Microbiol Ecol 62: 187-194.

He F, Legendre P. (1996). On species-area relations. Am Nat 148: 719-737.

He F, Gaston KJ. (2003). Occupancy, spatial variance, and the abundance of species. Am Nat 162: 366-375.

Huelsenbeck JP, Ronquist F. (2001). MRBAYES: Bayesian inference of phylogenetic trees. Bioinformatics 17: $754-755$

Hunt DE, David LA, Gevers D, Preheim SP, Alm EJ, Polz MF. (2008). Resource partitioning and sympatric differentiation among closely related bacterioplankton. Science 320: 1081-1085.

Huse SM, Huber JA, Morrison HG, Sogin ML, Welch DM. (2007). Accuracy and quality of massively parallel DNA pyrosequencing. Genome Biol 8: R143.

Huse SM, Welch DM, Morrison HG, Sogin ML. (2010). Ironing out the wrinkles in the rare biosphere through improved OTU clustering. Environ Microbiol 12: 1889-1898.

Jetten MSM, Stams AJM, Zehnder AJB. (1990). Acetate threshold values and acetate activating enzymes in methanogenic bacteria. Fems Microbiol Lett 73: 339-344.

Johnson ZI, Zinser ER, Coe A, McNulty NP, Woodward EM, Chisholm SW. (2006). Niche partitioning among Prochlorococcus ecotypes along ocean-scale environmental gradients. Science 311: 1737-1740.

Jorgensen BB. (1982). Mineralisation of organic matter in the sea-bed-the role of sulfate reduction. Nature $\mathbf{2 9 6}$ : 643-645.

Katoh K, Misawa K, Kuma K, Miyata T. (2002). MAFFT: a novel method for rapid multiple sequence alignment based on fast fourier transform. Nucleic Acids Res 30: 3059-3066.

Kuever J, Rainey FA, Widdel F. (2005). Desulfobulbus. In: Garrity GM (ed.). Bergey's Manual of Systematic Bacteriology. Volume 2: The Proteobacteria; Part $C$ The Alpha-Beta-, Delta and Epsilonproteobacteria. Springer: New York, pp 988-992.

Kunin V, Engelbrektson A, Ochman H, Hugenholtz P. (2010). Wrinkles in the rare biosphere: pyrosequencing errors can lead to artificial inflation of diversity estimates. Environ Microbiol 12: 118-123. 
Laanbroek HJ, Veldkamp H. (1982). Microbial interactions in sediment communities. Philos Trans $R$ Soc Lond $B$ Biol Sci 297: 533-550.

Li W, Godzik A. (2006). Cd-hit: a fast program for clustering and comparing large sets of protein or nucleotide sequences. Bioinformatics 22: 1658-1659.

Lien T, Madsen M, Steen IH, Gjerdevik K. (1998). Desulfobulbus rhabdoformis sp. nov., a sulfate reducer from a water-oil separation system. Int J Syst Bacteriol 48(Part 2): 469-474.

Lovley DR, Klug MJ. (1983). Sulfate reducers can out compete methanogens at freshwater sulfate concentrations. Appl Environ Microbiol 45: 187-192.

Lovley DR, Dwyer DF, Klug MJ. (1982). Kinetic analysis of competition between sulfate reducers and methanogens for hydrogen in sediments. Appl Environ Microbiol 43: 1373-1379.

Loy A, Lehner A, Lee N, Adamczyk J, Meier H, Ernst J et al. (2002). Oligonucleotide microarray for $16 \mathrm{~S}$ rRNA gene-based detection of all recognized lineages of sulfate-reducing prokaryotes in the environment. Appl Environ Microbiol 68: 5064-5081.

Lozupone C, Knight R. (2005). UniFrac: a new phylogenetic method for comparing microbial communities. Appl Environ Microbiol 71: 8228-8235.

Ludwig W, Strunk O, Westram R, Richter L, Meier H, Yadhukumar et al. (2004). ARB: a software environment for sequence data. Nucleic Acids Res 32: 1363-1371.

Macarthur RH. (1958). Population ecology of some warblers of northeastern coniferous forests. Ecology 39: 599-619.

Margulies M, Egholm M, Altman WE, Attiya S, Bader JS, Bemben LA et al. (2005). Genome sequencing in microfabricated high-density picolitre reactors. Nature 437: 376-380.

Martin AP. (2002). Phylogenetic approaches for describing and comparing the diversity of microbial communities. Appl Environ Microbiol 68: 3673-3682.

May RM, Macarthur RH. (1972). Niche Overlap as a Function of Environmental Variability. Proc Natl Acad Sci USA 69: 1109-1113.

Oakley BB, Carbonero F, van der Gast CJ, Hawkins RJ, Purdy KJ. (2010). Evolutionary divergence and biogeography of sympatric niche-differentiated bacterial populations. ISME J 4: 488-497.

Oakley BB, Dowd SE, Purdy KJ. (2011). ThermoPhyl: a software tool for selecting phylogenetically optimized conventional and quantitative-PCR taxon-targeted assays for use with complex samples. FEMS Microbiol Ecol 77: 17-27.

Oakley BB, Carbonero F, van der Gast CJ, Hawkins RJ, Purdy KJ. (2010). Evolutionary divergence and biogeography of sympatric niche-differentiated bacterial populations. ISME J 4: 488-497.

Oksanen J, Blanchet FG, Kindt R, Legendre P, O’Hara RB, Simpson GL et al. (2010). vegan: Community Ecology Package. $R$ package version 1.17-4.

Patel GB. (2001). Methanosaeta. In: Garrity GM (ed.). Bergey's Manual of Systematic Bacteriology. Volume 1: The Archaea and the Deeply Branching and Phototrophic Bacteria. Springer: New York, pp 289-294.

Patel GB, Sprott GD. (1990). Methanosaeta concilii gen. nov., sp. nov. ('Methanothrix concilii') and Methanosaeta thermoacetophila nom. rev., comb. nov. Int J Syst Bacteriol 40: 79-82.
Plugge CM, Plugge CM, Zhang W, Scholten JC, Stams AJ. (2011). Metabolic flexibility of sulfate-reducing bacteria. Front Microbiol 2: 1-8.

Purdy KJ. (2005). Nucleic acid recovery from complex environmental samples. Methods Enzymol 397: 271-292.

Purdy KJ, Hurd PJ, Moya-Laran J, Trimmer M, Oakley BB, Woodward G. (2010). Systems biology for ecology: from molecules to ecosystems. In: Guy W (ed.). Advances in Ecological Research. Academic Press: San Diego, USA, pp 87-149.

Purdy KJ, Embley TM, Takii S, Nedwell DB. (1996). Rapid Extraction of DNA and rRNA from Sediments by a Novel Hydroxyapatite Spin-Column Method. Appl Environ Microbiol 62: 3905-3907.

Purdy KJ, Nedwell DB, Embley TM, Takii S. (1997). Use of 16S rRNA-targeted oligonucleotide probes to investigate the occurrence and selection of sulphate-reducing bacteria in response to nutrient addition to sediment slurry microcosms from a Japanese estuary. FEMS Microbiol Ecol 24: 221-234.

$\mathrm{R}$ Development Core Team (2010). R: a language and environment for statistical computing. $\mathrm{R}$ Foundation for Statistical Computing: Vienna, Austria.

Rice P, Longden I, Bleasby A. (2000). EMBOSS: the European molecular biology open software suite. Trends Genet 16: 276-277.

Sass A, Sass A, Rütters H, Cypionka H, Sass H. (2002). Desulfobulbus mediterraneus sp. nov., a sulfate-reducing bacterium growing on mono- and disaccharides. Arch Microbiol 177: 468-474.

Schloss PD, Westcott SL, Ryabin T, Hall JR, Hartmann M, Hollister EB et al. (2009). Introducing mothur: open-source, platform-independent, communitysupported software for describing and comparing microbial communities. Appl Environ Microbiol 75: 7537-7541.

Stams AJM, Kremer DR, Weenk GH, Hansen TA. (1984). Pathway of propionate formation in Desulfobulbus propionicus. Arch Microbiol 139: 167-173.

Suzuki D, Ueki A, Amaishi A, Ueki K. (2007). Desulfobulbus japonicus sp. nov., a novel Gram-negative propionate-oxidizing, sulfate-reducing bacterium isolated from an estuarine sediment in Japan. Int $J$ Syst Evol Microbiol 57: 849-855.

Wagner M, Roger AJ, Flax JL, Brusseau GA, Stahl DA. (1998). Phylogeny of dissimilatory sulfite reductases supports an early origin of sulfate respiration. J Bacteriol 180: 2975-2982.

Ward DM, Winfrey MR. (1985). Interactions between methanogenic and sulfate-reducing bacteria in sediments In: Jannasch HW, Williams PJL (eds). Advances in Microbial Ecology. Plenum Press: New York, pp 219-286.

West NJ, Scanlan DJ. (1999). Niche-partitioning of Prochlorococcus populations in a stratified water column in the eastern North Atlantic Ocean. Appl Environ Microbiol 65: 2585-2591.

Whitman WB, Coleman DC, Wiebe WJ. (1998). Prokaryotes: the unseen majority. Proc Natl Acad Sci USA 95: 6578-6583.

Widdel F. (1988). Microbiology and ecology of sulfate- and sulfur-reducing bacteria, In: Zehnder AJB (ed.). Biology of Anaerobic Organisms. John Wiley \& Sons, Inc.: New York, pp 469-585. 